OPEN ACCESS

Edited by:

Miklos Fuzi,

Semmelweis University, Hungary

Reviewed by:

Nicola Petrosillo,

Istituto Nazionale per le Malattie Infettive Lazzaro Spallanzani (IRCCS),

Italy

Tommaso Giani,

University of Siena, Italy

*Correspondence:

Raffaele Zarrill

rafzarri@unina.it

Specialty section:

This article was submitted to

Antimicrobials, Resistance

and Chemotherapy,

a section of the journal

Frontiers in Microbiology

Received: 26 July 2017 Accepted: 19 October 2017 Published: 03 November 2017

Citation:

Esposito EP, Gaiarsa S,

Del Franco M, Crivaro V,

Bernardo M, Cuccurullo $S$,

Pennino F, Triassi M, Marone $P$,

Sassera D and Zarrilli R (2017) A Novel IncA/C1 Group Conjugative

Plasmid, Encoding VIM-1

Metallo-Beta-Lactamase, Mediates

the Acquisition of Carbapenem

Resistance in ST104 Klebsiella pneumoniae Isolates from Neonates

in the Intensive Care Unit of

V. Monaldi Hospital in Naples.

Front. Microbiol. 8:2135.

doi: 10.3389/fmicb.2017.02135

\section{A Novel IncA/C1 Group Conjugative Plasmid, Encoding VIM-1 Metallo-Beta-Lactamase, Mediates the Acquisition of Carbapenem Resistance in ST104 Klebsiella} pneumoniae Isolates from Neonates in the Intensive Care Unit of
V. Monaldi Hospital in Naples

\author{
Eliana P. Esposito', Stefano Gaiarsa ${ }^{2,3}$, Mariateresa Del Franco'1, Valeria Crivaro", \\ Mariano Bernardo ${ }^{4}$, Susanna Cuccurullo4, Francesca Pennino ${ }^{1}$, Maria Triassi' ${ }^{1}$, \\ Piero Marone ${ }^{3}$, Davide Sassera ${ }^{5}$ and Raffaele Zarrilli1,6*
}

'Department of Public Health, University of Naples 'Federico II', Naples, Italy, ${ }^{2}$ Department of Bioscience, University of Milan, Milan, Italy, ${ }^{3}$ Microbiology and Virology Unit, Fondazione Istituto di Ricovero e Cura a Carattere Scientifico (IRCCS) Policlinico San Matteo, Pavia, Italy, ${ }^{4}$ Azienda Ospedaliera di Rilievo Nazionale (AORN) dei Colli, V. Monaldi Hospital, Naples, Italy, ${ }^{5}$ Department of Biology and Biotechnologies, University of Pavia, Pavia, Italy, ${ }^{6}$ Centro di Inngegneria Genetica (CEINGE) Biotecnologie Avanzate, Naples, Italy

The emergence of carbapenemase producing Enterobacteriaceae has raised major public health concern. The aim of this study was to investigate the molecular epidemiology and the mechanism of carbapenem resistance acquisition of multidrugresistant Klebsiella pneumoniae isolates from 20 neonates in the neonatal intensive care unit (NICU) of the V. Monaldi Hospital in Naples, Italy, from April 2015 to March 2016. Genotype analysis by pulsed-field gel electrophoresis (PFGE) and multi-locus sequence typing (MLST) identified PFGE type $A$ and subtypes $A 1$ and A2 in 17, 2, and 1 isolates, respectively, and assigned all isolates to sequence type (ST) 104. K. pneumoniae isolates were resistant to all classes of $\beta$-lactams including carbapenems, fosfomycin, gentamicin, and trimethoprim-sulfamethoxazole, but susceptible to quinolones, amikacin, and colistin. Conjugation experiments demonstrated that resistance to third-generation cephems and imipenem could be transferred along with an IncA/C plasmid containing the extended spectrum $\beta$-lactamase blasHV-12 and carbapenem-hydrolyzing metallo- $\beta$-lactamase blavIM-1 genes. The plasmid that we called plncAC_KP4898 was 156,252 bp in size and included a typical IncA/C backbone, which was assigned to ST12 and core genome (cg) ST12.1 using the IncA/C plasmid MLST (PMLST) scheme. plncAC_KP4898 showed a mosaic structure with blavIM-1 into a class I integron, blasHV-12 flanked by IS6 elements, a mercury resistance and a macrolide $2^{\prime}$-phosphotransferase clusters, ant( $\left(3^{\prime \prime}\right)$, 
aph(3"), aacA4, qnrA1, sul1, and dfrA14 conferring resistance to aminoglycosides, quinolones, sulfonamides, and trimethoprim, respectively, several genes predicted to encode transfer functions and proteins involved in DNA transposition. The acquisition of plncAC_KP4898 carrying blavIM-1 and blasHV-12 contributed to the spread of ST104 K. pneumoniae in the NICU of V. Monaldi Hospital in Naples.

Keywords: carbapenemase producing Klebsiella pneumoniae, VIM-1 carbapenemase, IncA/C plasmid, horizontal gene transfer, neonatal intensive care unit

\section{INTRODUCTION}

The spread of carbapenem-resistant Enterobacteriaceae (CRE) has increased globally and these strains have become endemic in several countries including Italy. CRE may colonize or infect patients both in the hospital and in the community setting (Nordmann and Poirel, 2014; Del Franco et al., 2015; Onori et al., 2015; Pitout et al., 2015; Conte et al., 2016; Logan and Weinstein, 2017). The prevalence of CRE infections is increasing among children and neonates also (Logan, 2012; Logan et al., 2015; Zhu et al., 2015).

The international spread of CRE is primarily due to clonal expansion of isolates belonging to Klebsiella pneumoniae and Escherichia coli epidemic clonal lineages (Bialek-Davenet et al., 2014; Nordmann and Poirel, 2014; Del Franco et al., 2015; Gaiarsa et al., 2015; Pitout et al., 2015; Conte et al., 2016; Logan and Weinstein, 2017).

Additionally, CRE dissemination is contributed by horizontal gene transfer of carbapenemase genes carried by transposons and plasmids (Pitout et al., 2015; Logan and Weinstein, 2017). Class B metallo- $\beta$-lactamases (MBLs) (IMP, VIM, NDM), class A (KPC) or class D (OXA-48) serine carbapenemases have been isolated worldwide (Nordmann and Poirel, 2014; Del Franco et al., 2015; Pitout et al., 2015; Conte et al., 2016; Giani et al., 2017; Grundmann et al., 2017; Khan et al., 2017; Logan and Weinstein, 2017; Matsumura et al., 2017). Of these, MBLs show increasing clinical relevance because they cannot be neutralized by the available $\beta$-lactamase inhibitors and are able to horizontally disseminate via mobile genetic elements. Among acquired MBLs, VIM- and NDM-type enzymes are those having the widest geographical distribution and range of bacterial hosts (Di Pilato et al., 2014; Peirano et al., 2014; Zurfluh et al., 2015; Khan et al., 2017; Matsumura et al., 2017).

Because intestinal carriage of $\mathrm{CRE}$ is an important source of transmission, guidelines have been established worldwide to monitor and isolate CRE carriers in health care facilities (Nordmann and Poirel, 2014; Viau et al., 2016).

The aim of this study was to analyze the molecular epidemiology of VIM-1 producing $K$. pneumoniae isolates from intestinal carriers in neonatal intensive care unit (NICU) of an Italian hospital in Naples and to characterize the structure of the conjugative plasmid, which mediates the horizontal transfer of carbapenem resistance.

\section{MATERIALS AND METHODS}

\section{Setting}

The NICU of V. Monaldi Hospital in Naples is a tertiary care level NICU and consists of three rooms and 16 cot spaces with a 1:2-1:3 nurses/neonates ratio. The NICU serves approximately 260 admissions per year and admits exclusively babies from the regional Newborn Emergency Transport Service or Territorial Emergency Service and through the transfer from the internal departments of Pediatric Cardiology and Pediatric Heart Surgery. In case of necessity, the department performs repeated hospitalizations for some particular types of newborns (lower birth weight, heart disease, etc.). Active patient-based surveillance of healthcare-associated infections on neonates with $>2$ days NICU stay is performed as previously described (Horan et al., 2008; Crivaro et al., 2015). Surveillance of CRE in V. Monaldi Hospital, based on rectal swabs at hospital admission, is performed according to the guidelines of European Centre for Disease Prevention and Control [ECDC] (2012) and the Ministero della Salute (2013), Italy.

\section{Bacterial Strains and Microbiological Methods}

Klebsiella pneumoniae isolates were identified using the Vitek 2 automatic system and the ID-GNB card according to the manufacturer's instructions (bioMérieux, Marcy l'Etoile, France) as previously described (Del Franco et al., 2015).

\section{Antimicrobial Susceptibility Testing}

Carbapenem resistance of Enterobacteriaceae was screened using the meropenem disk alone as previously described (Pournaras et al., 2013). Identification of MBL activity was performed using imipenem + EDTA $15+750 \mu \mathrm{g}$ combined disk method (ROSCO Diagnostica A/S, Taastrup, Denmark). Susceptibility tests were performed using the Vitek 2 system and the AST-GN card (bioMérieux, Marcy l'Etoile, France); carbapenem and colistin susceptibility were evaluated by broth microdilution in MuellerHinton broth II (MHBII) according to Clinical and Laboratory Standards Institute guidelines (CLSI, 2015). Breakpoints values were those recommended by the EUCAST (2016).

\section{Molecular Analysis of Antimicrobial Resistance Genes}

Characterization of $\beta$-lactamase genes was performed as previously described (Poirel et al., 2011). Two multiplex PCRs were set up, the reaction no. 1 including detection of bla $\mathrm{KPC}$ 
and $b l a_{\text {OXA-48-like }}$ and the reaction no. 2 including detection of bla $a_{\mathrm{IMP}}, b l a_{\mathrm{VIM}}$, and bla $a_{\mathrm{NDM}}$. The following thermal cycling conditions were used: $3 \mathrm{~min}$ at $94^{\circ} \mathrm{C}$ and 35 cycles of amplification consisting of $1 \mathrm{~min}$ at $94^{\circ} \mathrm{C}, 1 \mathrm{~min}$ at $57^{\circ} \mathrm{C}$, and $1 \mathrm{~min}$ at $72^{\circ} \mathrm{C}$ with $5 \mathrm{~min}$ at $72^{\circ} \mathrm{C}$ for the final extension. PCR products were analyzed by electrophoresis in a $1.8 \%$ agarose gel stained with ethidium bromide. The following strains were used as positive quality controls: Acinetobacter baumannii AC 54/97 (Riccio et al., 2000), A. baumannii 161/07 (Bonnin et al., 2012), K. pneumoniae D001 (Pournaras et al., 2013), K. pneumoniae 1, K. pneumoniae 2, and E. coli 6 (Del Franco et al., 2015) for bla $a_{\mathrm{IMP}-2}, b l a_{\mathrm{NDM}-1}, b l a_{\mathrm{OXA}-48}, b l a_{\mathrm{KPC}-2}, b l a_{\mathrm{KPC}-3}$, and bla $a_{\mathrm{VIM}-1}$ carbapenemase genes, respectively. The full-length alleles of blavim were amplified using primers $5^{\prime}$ VIM1 and $3^{\prime}$ VIM1 shown in Supplementary Table S1. Sanger DNA sequencing and identification of deduced amino acid sequences were performed as previously described (Del Franco et al., 2015).

\section{PFGE Typing and Dendrogram Analysis}

Klebsiella pneumoniae isolates were genotyped by XbaI DNA macrorestriction, pulsed-field gel electrophoresis (PFGE) and dendrogram analysis as described previously (Del Franco et al., 2015).

\section{MLST Analysis}

Klebsiella pneumoniae isolates were typed using the Institut Pasteur's MLST (multi-locus sequence typing) scheme (Diancourt et al., 2005) and primes and PCR conditions available at http://bigsdb.pasteur.fr/klebsiella/primers_used.html eBURST analysis of profiles available at http://bigsdb.pasteur. fr/perl/bigsdb/bigsdb.pl?db=pubmlst_klebsiella_seqdef_public\& page $=$ profiles was performed as described previously (Feil et al., 2004). Minimum spanning trees of sequence types (STs) were built by Phyloviz using the goeBURST algorithm (Francisco et al., 2012).

\section{Conjugative Transfer of Carbapenem Resistance and Plasmid Typing}

Filter mating experiments were performed using sodium azide resistant E. coli J53 (Jacoby and Han, 1996) as recipient strain in the presence of either $5 \mu \mathrm{g} / \mathrm{ml}$ imipenem and $100 \mathrm{mg} / \mathrm{l}$ sodium azide or $50 \mu \mathrm{g} / \mathrm{ml}$ ampicillin and $100 \mu \mathrm{g} / \mathrm{ml}$ sodium azide. The frequency of transfer was calculated as the number of transconjugants divided by the number of surviving recipients as previously described (Zarrilli et al., 2005). Plasmids typing was performed by PCR-based replicon typing (PBRT) kit (Diatheva s.r.l., Fano, Italy) using previously described primers and conditions (Carattoli et al., 2005, 2006).

\section{Whole-Genome Sequencing and Plasmid Reconstruction}

DNA was extracted from the K. pneumoniae parental strain, and from the $E$. coli strain before and after transconjugation using a DNeasy Blood \& Tissue Kit according to the manufacturer's instructions (Qiagen, Milan, Italy). Whole genome sequencing was performed using an Illumina Miseq platform with a 2 by 250 paired-end run after Nextera XT paired-end library preparation. Sequencing reads from the $K$. pneumoniae parental strain and from the E. coli strain before the transconjugation obtained in this study were assembled using the software SPAdes (Bankevich et al., 2012) with accurate settings.

The BIGSdb genome database software (Jolley and Maiden, 2010) was used in the BIGSdb-Kp database ${ }^{1}$ to identify genes associated with virulence, heavy metal and drug resistance in K. pneumoniae 4898 genome sequence. In Silico detection of plasmids was performed using PlasmidFinder Web tool at https://cge.cbs.dtu.dk/services/PlasmidFinder/ as previously described (Carattoli et al., 2014). Sequencing reads of the E. coli transconjugant strain containing the plasmid were mapped to the assembled genome of the same E. coli sequenced prior to transconjugation, using the mapping software Bowtie2 (Langmead and Salzberg, 2012). All non-mapping reads were extracted and assembled using SPAdes (Bankevich et al., 2012) with accurate settings. Presence of the obtained contigs in the original $K$. pneumoniae genome was verified by blast-searching followed by manual analysis, i.e., all the newly obtained contigs that aligned with the $K$. pneumoniae assembly with an identity over $95 \%$ were kept. The co-linearity of the contigs was assessed using Bandage tool for visualizing assembly graphs (Wick et al., 2015). The connections indicated by Bandage were used as starting point for finishing experiments by PCR and Sanger sequencing to bridge the ends of contigs (see Supplementary Table S1 for a complete list of PCR primers used).

\section{Plasmid in Silico Typing and Annotation}

Plasmid MLST (PMLST) and core genome PMLST (cgPMLST) analysis of Inc A/C plasmid profiles available at https://pubmlst.org/bigsdb?db=pubmlst_plasmid_seqdef was performed as previously described (Hancock et al., 2017). The 28 IncA/C conserved genes from each PMLST profile were aligned using Muscle (Edgar, 2004). Unreliable positions were removed from each alignment using Gblocks (Castresana, 2000). All alignments were concatenated and used as input for a maximum likelihood phylogenetic analysis, which was performed with the software PhyML 3.0 (Guindon et al., 2010) using the GTR substitution model. PMLST minimum spanning trees were built by Phyloviz (Francisco et al., 2012). The gene annotation of the plasmid was performed using the software Prokka (Seemann, 2014) followed by accurate manual control, based on blast-searches against the nr-protein database. Inverted repeats were identified manually, based on the sequence and patterns found in Hancock (AAC 2017).

\section{Nucleotide Sequence Accession Numbers}

The genome sequences of K. pneumoniae 4898 and plasmid pIncAC-KP4898 have been deposited in the GenBank nucleotide database under accession numbers FWYI00000000.1 and KY882285, respectively.

\footnotetext{
${ }^{1}$ http://bigsdb.pasteur.fr/perl/bigsdb/bigsdb.pl?db=pubmlst_klebsiella_seqdef_ public\&page $=$ sequenceQuery
} 


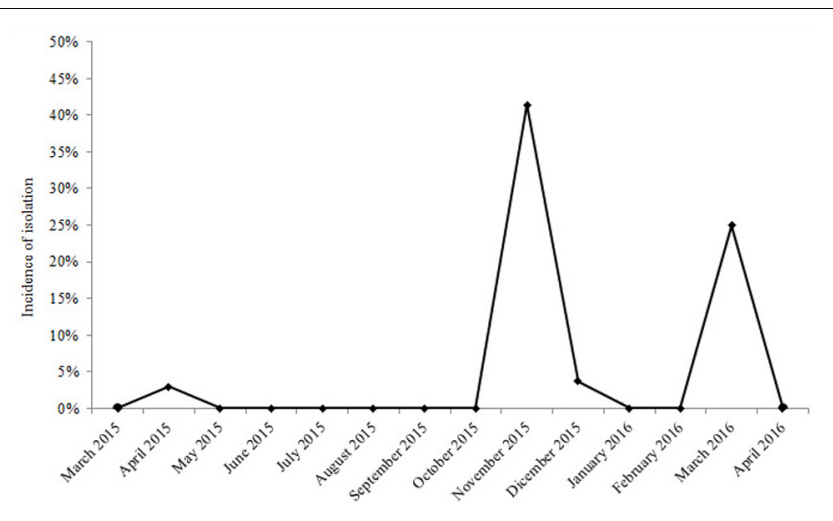

FIGURE 1 | Incidence of isolation of VIM-1 carbapenemase producing ST104 Klebsiella pneumoniae from neonates in the NICU of V. Monaldi Hospital in Naples, Italy, during 2015 and 2016.

\section{Ethics Statement}

The study has been evaluated by the local Ethics committee (Comitato Etico Università degli Studi della Campania "Luigi Vanvitelli” AOU "Luigi Vanvitelli” - AORN “Ospedali dei Colli”) (protocol number 421/2017). Because the patients included in the study were anonymized, no written informed consent was required.

\section{RESULTS}

\section{Epidemiology of CR K. pneumoniae in the NICU and Infection Control Measures}

The emergence of carbapenem-resistant (CR) K. pneumoniae was observed in the NICU of V. Monaldi Hospital from October 2015 to March 2016, when CR K. pneumoniae were isolated from rectal swabs of 19 neonates into two consecutive clusters. Only one sporadic CR K. pneumoniae isolate was obtained from a rectal swab of a neonate in the NICU of the hospital during the previous 22 months, while CRE were occasionally isolated from rectal swabs and other clinical specimens of adult patients in other wards of the hospital (Figure 1 and data not shown). Immediately following the first isolation of CR K. pneumoniae, a multimodal infection control program was implemented in the NICU, which included: weekly or biweekly screening for CRE at rectal swab of hospitalized neonates, increased frequency of environmental cleaning using chloride derivatives at $1100 \mathrm{ppm}$, reinforce handwashing compliance before and after patient contact, and daily visits to the ward by the hospital Infection Control Nurse. Colonized neonates were isolated either structurally or following strict adherence to contact precautions and staff cohorting was performed. During the two clusters, the ward was temporarily closed to external admissions. Environmental microbiological investigation of room surfaces, equipment, and staff hands failed to identify sources or reservoirs of CR K. pneumoniae. Neonates' mothers were not screened for the presence of CR K. pneumoniae. Because all neonates with CR $K$. pneumoniae isolation did not show signs of infection, they did not receive antimicrobial therapy against CR K. pneumoniae and were not eradicated before discharge. The cluster ended on March 2016 when the last CR K. pneumoniae intestinal carrier neonate was discharged from the ward (Figure 1).

\section{Antimicrobial Susceptibility Testing and Characterization of Carbapenemase Genes in CR K. pneumoniae Isolates}

All CR K. pneumoniae isolates from neonates in the NICU showed a MDR phenotype. In fact, they exhibited resistance or intermediate resistance to carbapenem (imipenem, meropenem, ertapenem), resistance to aminopenicillins, ureidopenicillins, third and fourth generation of cephalosporins (ceftazidime, cefotaxime, cefepime), gentamicin, fosfomycin, and trimethoprim-sulfamethoxazole, but were susceptible to amikacin, ciprofloxacin, and colistin (Table 1). All K. pneumoniae isolates gave a positive result in the MBL-assay performed using the imipenem-EDTA combined disk method. PCR and sequence analysis identified the presence of bla VIM-1 $_{1}$ but not any of the other carbapenemase genes tested in all CR K. pneumoniae isolates from NICU (Figure 2).

\section{Molecular Epidemiology of CR K. pneumoniae}

To assess whether the increase of CR K. pneumoniae isolation in the NICU was caused by the spread of epidemic strains, all 20 CR K. pneumoniae isolates from 20 neonates were genotyped by $\mathrm{XbaI}$ digestion, PFGE, and dendrogram analysis. Molecular analysis identified an identical macrorestriction pattern in 17 isolates, which we named PFGE type A, whereas two isolates showing difference in the migration of one band and one isolate

TABLE 1 | Antibiotic susceptibility profiles of MDR K. pneumoniae in the NICU.

\begin{tabular}{lccc}
\hline \multicolumn{3}{c}{ MIC* $^{*}(\mathbf{m g} / \mathbf{l})$} & \\
\hline Antibiotic & \multicolumn{2}{c}{ K. pneumoniae (20 total stains) } \\
\cline { 2 - 4 } & MIC $_{\mathbf{5 0}}$ & $\mathbf{M I C}_{\mathbf{9 0}}$ & Range \\
\hline Amoxicillin & $>32$ & $>32$ & $>32$ \\
Piperacillin-tazobactam & $>128$ & $>128$ & $>128$ \\
Ceftazidime & $>64$ & $>64$ & $>64$ \\
Cefotaxime & $>64$ & $>64$ & $>64$ \\
Cefepime & $>64$ & $>64$ & 32 to $>64$ \\
Imipenem & 8 & $>16$ & 8 to $>16$ \\
Meropenem & $>16$ & $>16$ & $>16$ \\
Ertapenem & 4 & 4 & 4 \\
Fosfomycin & $>128$ & $>128$ & $>128$ \\
Amikacin & $\leq 2$ & $\leq 2$ & $\leq 2$ \\
Gentamicin & 4 & 4 & 4 \\
Ciprofloxacin & 0.5 & 1 & $0.5-1$ \\
Trimethoprim-sulfamethoxazole & $>320$ & $>320$ & $>320$ \\
Tigecycline & $\leq 0.5$ & $\leq 0.5$ & $\leq 0.5$ \\
Colistin & $\leq 0.5$ & $\leq 0.5$ & $\leq 0.5$ \\
\hline
\end{tabular}

*Minimal inhibitory concentration. 


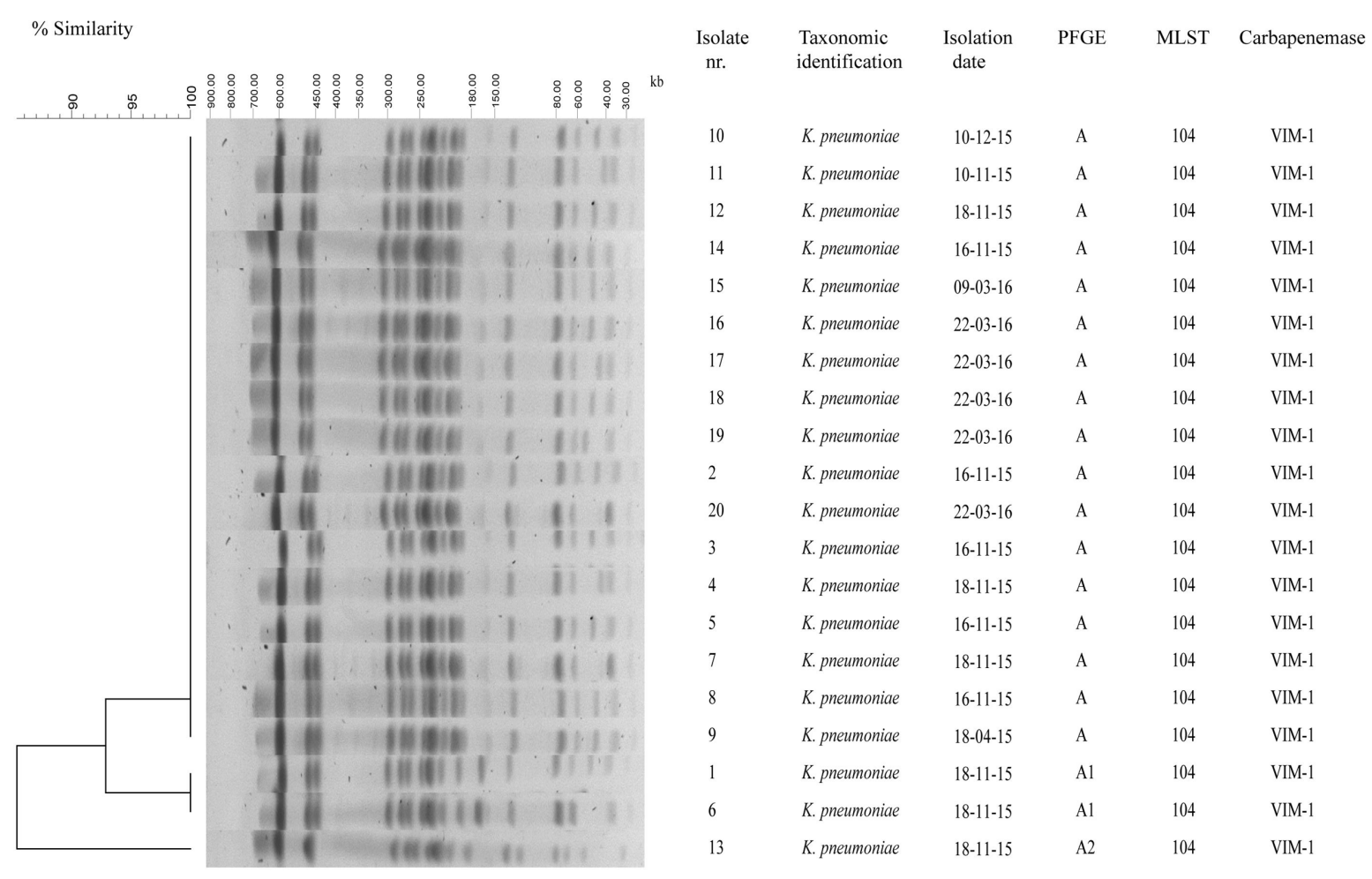

FIGURE 2 | Genotypic analysis of VIM-1 carbapenemase producing K. pneumoniae isolates in the NICU. Dendrogram analysis of Xbal PFGE profiles of VIM-1 carbapenemase producing K. pneumoniae isolates from the NICU. Percentage of similarity and sizes in kilobase (kb) of lambda DNA molecular mass markers are indicated. Isolate number, taxonomic identification, isolation date, PFGE type, MLST, and carbapenemase are shown also.

in the migration of four bands and a similarity of $>85 \%$ at dendrogram analysis were classified into subtypes A1 and A2, respectively (Figure 2). The above data indicated that the increase of CR K. pneumoniae isolation in the NICU was caused by the spread of a single VIM-1-producing K. pneumoniae epidemic genotype. MLST analysis assigned CR K. pneumoniae isolates with PFGE type A and subtypes A1 and A2 to ST104 (Figure 2). K. pneumoniae ST104 isolate from milk during bovine mastitis and K. pneumoniae ST1923 and ST1942 isolates from human blood and human feces, respectively, which were single-locus variants of ST104, and 21 other STs, which were double-locus variants of ST104, were reported worldwide and found in Klebsiella PubMLST isolates database ${ }^{2}$ (Figure 3).

\section{Genomic Features of VIM-1-Producing K. pneumoniae ST104 KP4898}

Additional epidemiological information was provided by genome sequence of representative K. pneumoniae KP4898 isolate. Genome sequence confirmed MLST assignment of K. pneumoniae KP4898 isolate to ST104. Capsular typing through sequencing of $\mathrm{CD} 1-\mathrm{VR} 2-\mathrm{CD} 2$ region of $\mathrm{wZC}$ and outer membrane protein wzi genes of locus identified wzc-32 and wzi-102 alleles, respectively, which are associated with

${ }^{2}$ http://bigsdb.pasteur.fr/perl/bigsdb/bigsdb.pl?db=pubmlst_klebsiella_isolates_ public\&page $=$ profiles
K31 capsular type. In the KP4898, an array of virulenceassociated genes was found, which includes the type- 3 fimbriae cluster $m r k A B C D F$ and transcription regulators $m r k H I J$, the yersiniabactin siderophore cluster $y b t A P Q S T U X$, the yersiniabactin receptor $f y u A$, yersiniabactin biosynthetic protein genes irp 1 and irp2 and allB and allD genes of allantoinase cluster. The analysis of drug-associated resistance genes in KP4898 genome identified bla $a_{\mathrm{SHV}-5}$ and bla $a_{\mathrm{SHV}-12}$ extendedspectrum $\beta$-lactamases, bla $a_{\mathrm{VIM}-1}$ carbapenemase, aadA1, aphA15, and aacA4 aminoglycoside resistance genes, a macrolide $2^{\prime}$-phosphotransferase cluster, other antimicrobial resistance genes, heavy metal resistance genes, efflux system, and regulators genes (Supplementary Table S2). The PlasmidFinder web tool identified $\mathrm{A} / \mathrm{C}, \mathrm{FII}(\mathrm{K})$ and $\mathrm{FIB}(\mathrm{K})$ replicons, thus suggesting the presence of at least three plasmids in KP4898 genome.

\section{Conjugal Transfer of Carbapenem Resistance}

The transfer of carbapenem resistance from VIM-1-producing ST104 K. pneumoniae isolates with PFGE type A and subtypes A1 and A2 was evaluated by filter mating experiments. Resistance or intermediate resistance to aminopenicillins, ureidopenicillins, third and fourth generation cephems, imipenem, but not meropenem and ertapenem, was transferred from ST104 K. pneumoniae isolates with PFGE type A and subtypes A1 and A2 to $E$. coli $\mathrm{J} 53$ aziR at frequency ranging from $6.5 \times 10^{-3}$ 


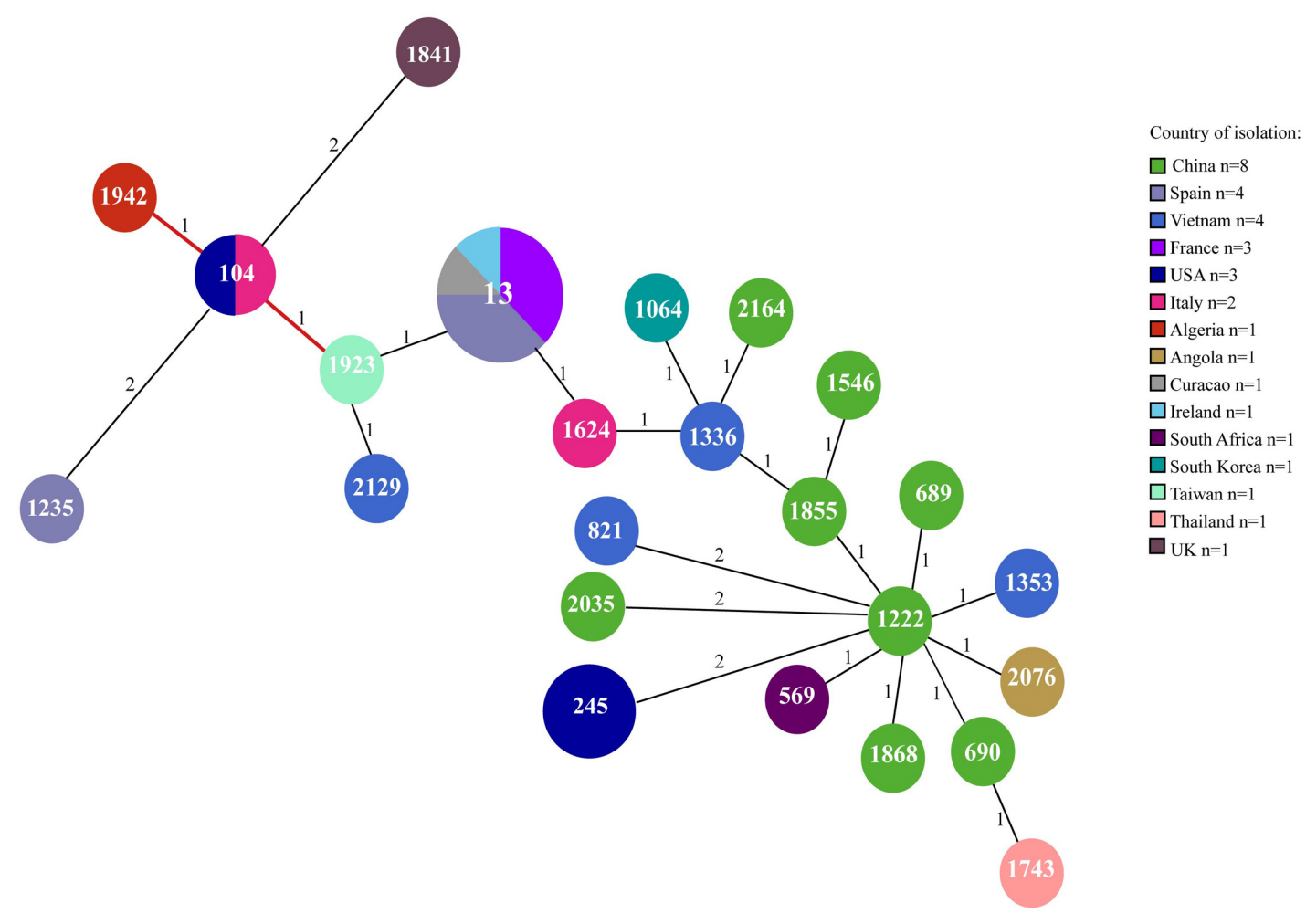

FIGURE 3 | Minimum spanning tree of ST104 and single-locus and double-locus variant isolates at Klebsiella PubMLST (http://bigsdb.pasteur.fr/perl/bigsdb/bigsdb. pl?db=pubmlst_klebsiella_isolates_public\&page=profiles). ST1942 and ST1923 are single-locus variant of ST104. The other STs are ST104 double-locus variant. Numbers inside each circle indicate the ST types. The number on the branch indicates the different alleles between STs. Circle size is proportional to the number of isolates belonging to the same ST type. The pie charts for each ST indicate the country of isolation.

to $1.5 \times 10^{-3} \mathrm{cfu} /$ recipient cells. The frequency of transfer of imipenem resistance from K. pneumoniae ST104/PFGE A, A1, and A2 isolates to E. coli J53 aziR did not change if transconjugants were selected in the presence of imipenem and sodium azide or ampicillin and sodium azide. All transconjugants showed identical antimicrobial susceptibility profile and resistance to sodium azide (Table 2). Moreover, all transconjugants showed a PFGE profile identical with that of the recipient strain. The presence of bla $a_{\mathrm{VIM}-1}$ and bla $a_{\mathrm{SHV}-12}$ genes was demonstrated in all transconjugants. Furthermore, $\mathrm{PBRT}$ identified $\mathrm{A} / \mathrm{C}$ replicon and IncA/C incompatibility group plasmid/s in ESBL positive and CR K. pneumoniae ST104 donor isolates and $E$. coli transconjugants expressing bla $a_{\mathrm{VIM}-1}$ and bla $a_{\text {SH-12 }}$ genes. The above data suggested that conjugative plasmid/s mediated the horizontal transfer of carbapenem resistance.

\section{Genetic Structure of plncAC-KP4898}

In the genome sequence of $E$. coli transconjugants, one single plasmid was identified, which was present in K. pneumoniae KP4898 donor strain, but not in E. coli J53 recipient strain, and was designated pIncAC-KP4898, for plasmid of IncA/C incompatibility group from $K$. pneumoniae 4898 isolate. The pIncAC-KP4898 plasmid was 156,252 bp in size, with an average $\mathrm{G}+\mathrm{C}$ content of $52.6 \%$. Genome annotation identified 190 open reading frames (ORFs), of which 145 were transcribed in a clockwise orientation, while the remaining 45 were transcribed counterclockwise. Of these ORFs, 14 were associated with plasmid DNA replication and partition, 16 with DNA transfer, 13 with DNA-restriction and site-specific DNA methylation, 25 with DNA transposition, 19 with antimicrobial resistance, and 103 with unknown functions (Figure 4 and Supplementary Table S3). The pIncAC-KP4898 scaffold included the $\operatorname{rep} A$, parA, parB, and 053 genes, parM, $k f r A$, and a putative toxin-antitoxin system, which were demonstrated to be important for maintenance and replication of IncA/C plasmid (Hancock et al., 2017). Based on RepA similarity (Carattoli et al., 2006), the pIncACKP4898 plasmid belongs to IncA/C1 group. The IncA/C PMLST scheme based on the repA, parA, parB, and 053 genes and the cgPMLST scheme that extend the 4-gene PMLST to 28 conserved genes (Hancock et al., 2017) assigned pIncAC-KP4898 to novel profiles, which corresponded to ST12 and ST12.1, respectively. The phylogeny of IncA/C 28-gene profiles showed that ST12.1 was more closely related to ST11.1, to which IncA/C1 group pRA1 plasmid (GenBank accession number FJ705807.1) has been assigned, than to the remaining 10 profiles (ST1 to ST10), which corresponded to IncA/C2 group plasmids (Figure 5).

Plasmid pIncAC-KP4898 contained a composite transposon of 25,716 bp (residues 139,120-156,252 and 1-8,583, G + C content: $57.7 \%$ ) with four IS6 family transposase and $14 \mathrm{bp}$ 
TABLE 2 | Conjugative transfer of carbapenem resistance.

\begin{tabular}{|c|c|c|c|}
\hline \multicolumn{4}{|c|}{ MIC (mg/l) } \\
\hline \multirow[t]{2}{*}{ Antibiotic } & \multicolumn{3}{|c|}{ Strain } \\
\hline & $\begin{array}{l}\text { K. pneumoniae } \\
\text { ST104/A-A2 }\end{array}$ & $\begin{array}{c}\text { E. coli J53 aziR } \\
\text { (p1KPST104) }\end{array}$ & $\begin{array}{c}\text { E. coli J53 } \\
\text { aziR }\end{array}$ \\
\hline Amoxicillin & $>32$ & $>32$ & 4 \\
\hline $\begin{array}{l}\text { Piperacillin- } \\
\text { tazobactam }\end{array}$ & $>128$ & $>128$ & 2 \\
\hline Ceftazidime & $>64$ & 8 & 0.25 \\
\hline Cefotaxime & $>64$ & $>64$ & $\leq 0.25$ \\
\hline Cefepime & $>64$ & 2 & $\leq 0.25$ \\
\hline Imipenem & 8 & 8 & 0.25 \\
\hline Meropenem & $>16$ & $\leq 0.25$ & $\leq 0.25$ \\
\hline Ertapenem & 4 & $\leq 0.5$ & $\leq 0.5$ \\
\hline Fosfomycin & $>128$ & $\leq 16$ & $\leq 16$ \\
\hline Amikacin & $\leq 2$ & $\leq 2$ & $\leq 2$ \\
\hline Gentamicin & 4 & $\leq 1$ & $\leq 1$ \\
\hline Ciprofloxacin & 0.5 & $\leq 0.25$ & $\leq 0.25$ \\
\hline $\begin{array}{l}\text { Trimethoprim- } \\
\text { sulfamethoxazole }\end{array}$ & $>320$ & $\leq 20$ & $\leq 20$ \\
\hline Tigecycline & $\leq 0.5$ & $\leq 0.5$ & $\leq 0.5$ \\
\hline Colistin & $\leq 0.5$ & $\leq 0.5$ & $\leq 0.5$ \\
\hline
\end{tabular}

inverted repeats (TTTGCAACAGTGCC) at residues 152,072152,086 and 8,569-8,583 ( $3^{\prime}$-flanking region) (Figure 4). Within this transposon lied a class 1 integron containing a $5^{\prime}$-conserved structure (CS) with int1 site-specific integrase, and five headto-tail arranged gene cassettes consisting of the genes bla $a_{\mathrm{VIM}-1}$, aacA4 family aminoglycoside $\mathrm{N}\left(6^{\prime}\right)$-acetyltransferase gene, aminoglycoside $O$-phosphotransferase $A P H\left(3^{\prime}\right)-X V$ encoding gene, $\operatorname{ANT}\left(3^{\prime \prime}\right)$ family aminoglycoside nucleotidyl transferase gene, type B-2 chloramphenicol O-acetyltransferase catB2 gene. The $3^{\prime}$-CS showed the qacED1-encoding gene, which confers resistance to quaternary ammonium compounds. The Tn3-like composite transposon included also the bla $a_{\mathrm{SHV}-12}$ gene flanked by IS6 family transposases in inverted orientation, a macrolide $2^{\prime}$-phosphotransferase gene cluster consisting of macrolide $2^{\prime}$-phosphotransferase $m p h(A)$, transporter $m f s$ and macrolide $2^{\prime}$-phosphotransferase I repressor A $m p h R$ genes, quinolone resistance pentapeptide repeat protein qnrAl gene, and sulfonamide-resistant dihydropteroate synthase sul1 genes. A mercury resistance gene clusters and trimethoprim-resistant dihydrofolate reductase $d f r A 14$ gene were carried by pIncACKP4898 apart from the identified transposon region. In addition, 16 genes encoding transfer functions and a conjugative apparatus were found in pIncAC-KP4898, which indicated that the plasmid was self-conjugative (Figure 4 and Supplementary Table S3).

\section{DISCUSSION}

The emergence of multidrug-resistant and carbapenem-resistant microorganisms has become an alarming phenomenon in children and neonates (Logan, 2012; Logan et al., 2015). Intestinal carriage of carbapenemase producing Enterobacteriaceae is an important reservoir and source of dissemination of resistance to carbapenems among Gram-negative bacteria in the community and in the hospital setting (Nordmann and Poirel, 2014; Viau et al., 2016; Logan and Weinstein, 2017).

The current study investigates the molecular epidemiology and the genetic mechanism of acquisition of carbapenem resistance of multidrug-resistant K. pneumoniae isolated into two consecutive clusters from rectal swabs of 20 neonates in the NICU of the V. Monaldi Hospital in Naples, Italy. Our data showed the selection of a single VIM-1-producing K. pneumoniae epidemic genotype assigned to PFGE type A, A1, and A2 and ST104, which was isolated only from patients in the NICU but not in other wards of the hospital, thus suggesting that crosstransmission among intestinal carrier neonates may have been favored the spread of VIM-1 producing $K$. pneumoniae epidemic clone. The diffusion of VIM-1 producers $K$. pneumoniae is uncommon in Italy, where the vast majority of carbapenemase producers K. pneumoniae are KPC producers (Giani et al., 2017; Grundmann et al., 2017). After the first isolation of ST104 K. pneumoniae from milk during bovine mastitis (Paulin-Curlee et al., 2007), sporadic isolations of ST104 (Baraniak et al., 2013; Maatallah et al., 2014; Esteban-Cantos et al., 2017), were reported from human infections/colonizations. Also, K. pneumoniae ST1923 and ST1942, which were single-loci variants of ST104, were isolated from human blood and human feces (Yan et al., 2015), and K. pneumoniae isolates assigned to 21 other STs, which were double-locus variants of ST104, were isolated from difference sources including clinical specimens (Figure 3). Since ST104 K. pneumoniae human isolates were either ESBL producing (Baraniak et al., 2013; Maatallah et al., 2014; EstebanCantos et al., 2017) or carbapenemase producing (EstebanCantos et al., 2017), we can hypothesize that antimicrobial resistance might have selected ST104 K. pneumoniae isolates among bacterial population.

In accordance with this, data reported herein showed that $K$. pneumoniae isolates from neonates in the NICU showed a MDR phenotype, being resistant to all classes of $\beta$-lactams including third and fourth generation cephems and carbapenems, fosfomycin, gentamicin, and trimethoprimsulfamethoxazole, but susceptible to quinolones, amikacin and colistin. This is particularly alarming in neonates, for which limited options of antimicrobial therapy are available. Also, genome sequence of VIM-1-producing K. pneumoniae ST104 KP4898 showed the presence of virulence-associated genes and antimicrobial resistance genes (Supplementary Table S2). Interestingly, ST104 K. pneumoniae isolates from neonates in the NICU showed resistance to antimicrobials frequently used in neonates, such as third and fourth generation cephems, carbapenems, gentamicin, and trimethoprim-sulfamethoxazole, while they retain susceptibility to fluoroquinolones, which are not recommended in this clinical setting. Since it has been demonstrated that previous combination antimicrobial treatment with ampicillin and gentamicin is independent risk factors for acquisition of extended-spectrum $\beta$-lactamaseproducing K. pneumoniae and Serratia marcescens in neonates (Crivaro et al., 2007), we can speculate that resistance to third and 


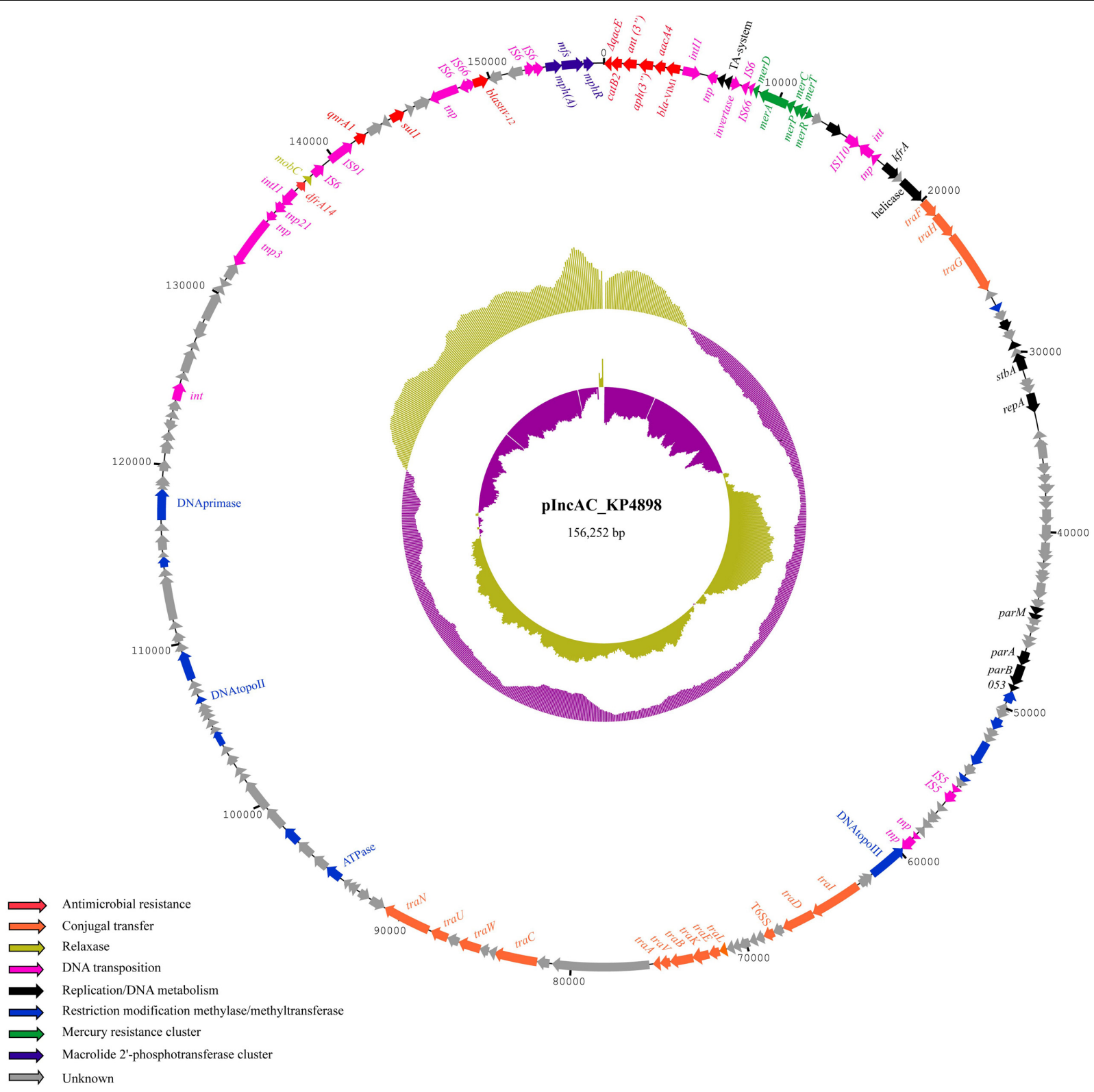

FIGURE 4 | Schematic map of plncAC_KP4898 plasmid. Circular map of plncAC_KP4898. The outermost ring represents the plncAC_KP4898 plasmid with arrows indicating genes/ORFs involved in the various cellular pathways. Functions of the genes and ORFs found are color coded and shown in the left bottom corner of the figure. The middle ring and the innermost ring represent a GC plot and a GC skew plot, respectively. For both plots, magenta and olive green indicate the measures below and above the average, respectively.

fourth generation cephems and carbapenems might have been selected by their frequent use in the NICU.

Several studies demonstrate that the horizontal gene transfer through conjugative plasmids and transposons contributes to the spread of resistance to carbapenems (Bialek-Davenet et al., 2014; Nordmann and Poirel, 2014; Viau et al., 2016). Accordingly, we showed that resistance to aminopenicillins, ureidopenicillins, third and fourth generation cephems, imipenem, but not meropenem and ertapenem, was transferred from VIM-1producing ST104 K. pneumoniae isolates assigned to either
PFGE type A, A1, or A2 to susceptible E. coli along with a blaVIM-1 positive plasmid of IncA/C1 incompatibility group and 156,252 bp in size, which we named PIncAC_KP4898 (Figure 4). Based on the above data, we postulate that imipenem resistance depends mainly on the expression of VIM-1 carbapenemase carried by pIncAC_KP4898, while meropenem and ertapenem resistance might be contributed by additional resistance mechanisms in K. pneumoniae isolates, such as altered permeability due to changes in the expression of porins or efflux systems (Goodman et al., 2016). Further experiments 


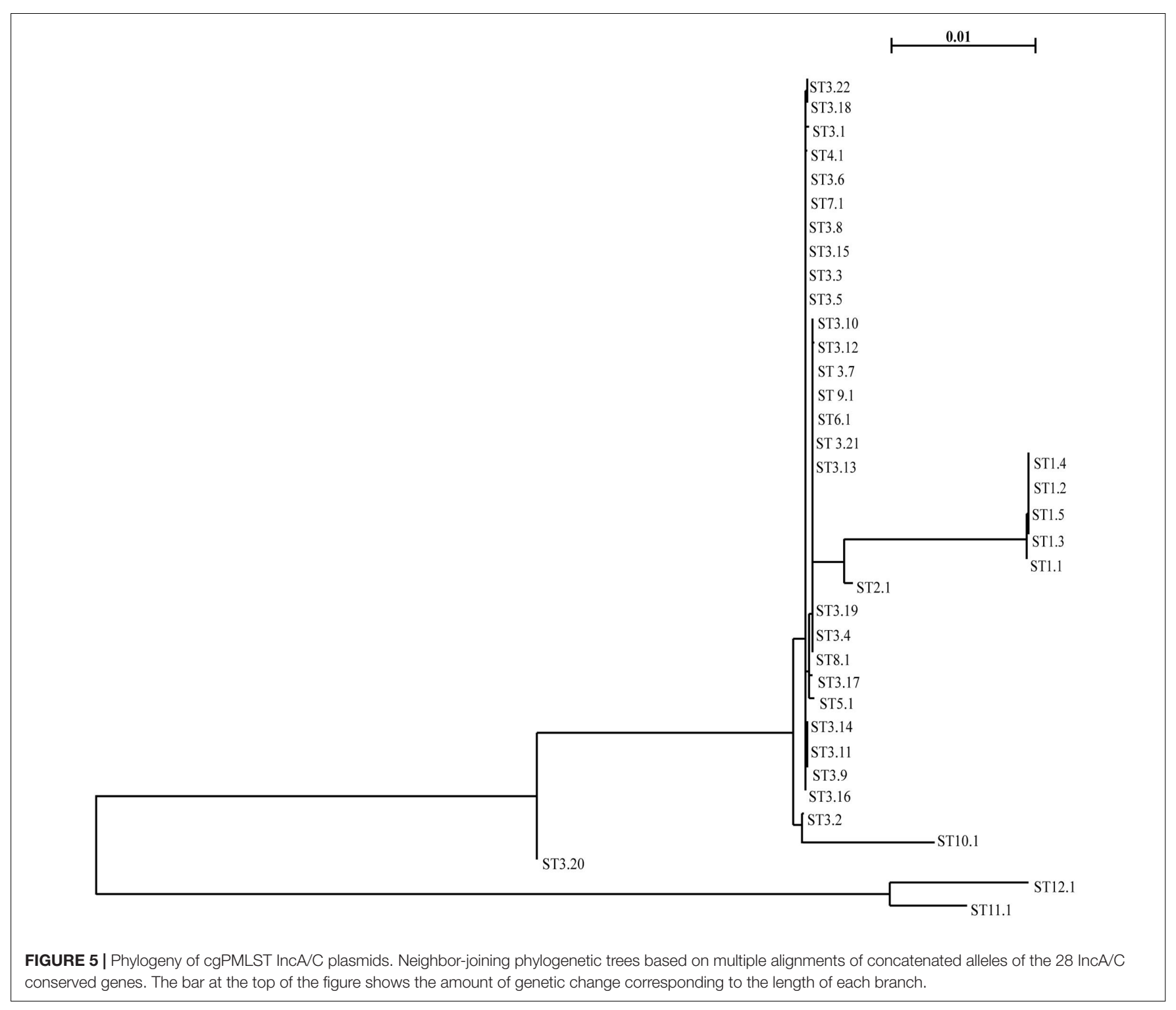

will be necessary to validate such hypothesis. The pIncACKP4898 scaffold was assigned to novel ST12 and ST12.1 profiles. Interestingly, it resulted to be more closely related to IncA/C1 group pRA1 plasmid from the fish pathogen Aeromonas hydrophila (Fricke et al., 2009) assigned to ST11.1, than to the majority of IncA/C plasmid belonging to IncA/C2 group and ST1 to ST10 profiles (Figure 5 and Supplementary Figure S1), which are associated with the carriage of $b l a_{\mathrm{NDM}}$ or $b l a_{\mathrm{CMY}}$ (Hancock et al., 2017). While InCA/C2 group plasmids were frequently found in Enterobacteriaceae isolated from human and non-human sources (Hancock et al., 2017), only two IncA/C1 group complete plasmid sequences are available in GenBank, pRA1 plasmid from A. hydrophila environmental isolate (Fricke et al., 2009) and pIncAC_KP4898 plasmid from ST104 K. pneumoniae clinical isolate described in this study. Of these, pIncAC_KP4898 is the first InCA/C1 plasmid carrying $b l a_{\text {VIM-like }}$ sequences. Similarly to IncA/C1 group pRA1 plasmid
(Fricke et al., 2009) and several IncA/C 2 group plasmids (Hancock et al., 2017), pIncAC-KP4898 carried several tra genes encoding transfer functions and was self-conjugative.

pIncAC_KP4898 plasmid presents a composite transposon of approximately $26 \mathrm{~kb}$, which includes bla $a_{\mathrm{SHV}-12}$ extended spectrum $\beta$-lactamase gene, flanked by IS6 elements and a class I integron with $b l a_{\mathrm{VIM}-1}$ carbapenemase gene, aac 44 family aminoglycoside $\mathrm{N}\left(6^{\prime}\right)$-acetyltransferase gene, aminoglycoside $O$-phosphotransferase $A P H\left(3^{\prime}\right)-X V$ encoding gene, $\operatorname{ANT}\left(3^{\prime \prime}\right)$ family aminoglycoside nucleotidyl transferase gene, type B-2 chloramphenicol $\mathrm{O}$-acetyltransferase catB2 gene. A class I integron showing identical gene cassettes array is found in $\mathrm{IncN}$ plasmid pOW16C2 from $K$. pneumoniae environmental isolate (Zurfluh et al., 2015), and non-typeable plasmids pAX22 from Achromobacter xylosoxidans (Di Pilato et al., 2014) and plasmid of $>300 \mathrm{~kb}$ from E. coli strain W1058 (GenBank accession number KF856617; Porres-Osante et al., 2014). Based on the 
above all data, we can postulate that genetic structure of class I integron with bla VIM-1 carried by pIncAC-KP4898 might have an environmental source. In accordance with our data, VIM1 has been shown to be the most prevalent allele variants among VIM-producing isolates, having global geographical distribution and being isolated in multiple Enterobacteriaceae species (Matsumura et al., 2017). Moreover, Aeromonas caviae carrying bla $a_{\mathrm{VIM}-1}$ and bla $a_{\mathrm{VIM}-35}$ inside class I integrons were isolated from clinical surveillance cultures in Israeli hospitals (Adler et al., 2014); A. caviae carrying bla $a_{\mathrm{VIM}-1}$ and bla $a_{\mathrm{SHV}-12}$ into a transferable plasmid were isolated from the blood cultures of 1-day-old newborn in Florence, Italy (Antonelli et al., 2016). A macrolide $2^{\prime}$-phosphotransferase gene cluster inside the composite transposon and a mercury resistance gene clusters and trimethoprim-resistant dihydrofolate reductase dfrA14 gene outside the transposon region were additional resistance genes, which might have contributed to the selection of pIncAC_KP4898 into ST104 K. pneumoniae.

\section{CONCLUSION}

The spread of carbapenem resistance in $K$. pneumoniae from neonates in the NICU was due to the acquisition of plasmid pIncAC-KP4898, carrying the bla $a_{\mathrm{VIM}-1}$ gene and several additional resistance genes into the scaffold of an IncA/C1 group self-conjugative plasmid. The composite genetic structure of pIncAC-KP4898 might have been generated by the acquisition of different regions from different sources mediated by multiple recombination events.

\section{AUTHOR CONTRIBUTIONS}

DS and RZ conceived the study and participated in its design and coordination. VC collected the epidemiological data and

\section{REFERENCES}

Adler, A., Assous, M. V., Paikin, S., Shulman, A., Miller-Roll, T., Hillel, S., et al. (2014). Emergence of VIM-producing Aeromonas caviae in Israeli hospitals. J. Antimicrob. Chemother. 69, 1211-1214. doi: 10.1093/jac/dkt505

Antonelli, A., D’Andrea, M. M., Montagnani, C., Bartolesi, A. M., Di Pilato, V., Fiorini, P., et al. (2016). Newborn bacteraemia caused by an Aeromonas caviae producing the VIM-1 and SHV-12 $\beta$-lactamases, encoded by a transferable plasmid. J. Antimicrob. Chemother. 71, 272-274. doi: 10.1093/jac/dkv304

Bankevich, A., Nurk, S., Antipov, D., Gurevich, A. A., Dvorkin, M., Kulikov, A. S., et al. (2012). SPAdes: a new genome assembly algorithm and its applications to single-cell sequencing. J. Comput. Biol. 19, 455-477. doi: 10.1089/cmb.2012. 0021

Baraniak, A., Izdebski, R., Fiett, J., Sadowy, E., Adler, A., Kazma, M., et al. (2013). Comparative population analysis of Klebsiella pneumoniae strains with extended-spectrum $\beta$-lactamases colonizing patients in rehabilitation centers in four countries. Antimicrob. Agents Chemother. 57, 1992-1997. doi: 10.1128/ AAC.02571-12

Bialek-Davenet, S., Criscuolo, A., Ailloud, F., Passet, V., Jones, L., DelannoyVieillard, A. S., et al. (2014). Genomic definition of hypervirulent and multidrug-resistant Klebsiella pneumoniae clonal groups. Emerg. Infect. Dis. 20, 1812-1820. doi: 10.3201/eid2011.140206

Bonnin, R. A., Poirel, L., Naas, T., Pirs, M., Seme, K., Schrenzel, J., et al. (2012). Dissemination of New Delhi metallo- $\beta$-lactamase-1-producing Acinetobacter performed the infection control in the hospital. MB and SC collected the microbiological data. EE, MDF, FP, and MT performed laboratory analyses. SG, PM, DS, and RZ performed data analyses. EE, SG, DS, and RZ wrote the manuscript. All authors read and approved the final manuscript.

\section{FUNDING}

This work was supported in part by grant from University of Naples "Federico II" (Fondo d'Ateneo per la Ricerca to MT and RZ).

\section{ACKNOWLEDGMENTS}

We thank Erika Scaltriti, Istituto Zooprofilattico Sperimentale della Lombardia e dell'Emilia-Romagna, Parma, Italy, for technical assistance in whole genome sequencing. We thank also Dr. Steven Hancock, University of Queensland, Brisbane, Australia, for curating plasmid MLST data and making them publicly available at Plasmid MLST Databases (https://pubmlst. org/plasmid/). RZ dedicates this study to the memory of Stella Carlomagno, a mentor in bacterial genetic and life sciences.

\section{SUPPLEMENTARY MATERIAL}

The Supplementary Material for this article can be found online at: https://www.frontiersin.org/articles/10.3389/fmicb. 2017.02135/full\#supplementary-material

FIGURE S1 | Minimum spanning tree showing the IncA/C core gene PMLST (cgPMLST). Numbers inside each circle are the cgST types. The size of the circle indicates the number of the isolates belonging to the same cgST type. The number on the branch indicates the different alleles between cgSTs.

baumannii in Europe. Clin. Microbiol. Infect. 18, E362-E365. doi: 10.1111/j. 1469-0691.2012.03928.x

Carattoli, A., Bertini, A., Villa, L., Falbo, V., Hopkins, K. L., and Threlfall, E. J. (2005). Identification of plasmids by PCR-based replicon typing. J. Microbiol. Methods 63, 219-228. doi: 10.1016/j.mimet.2005.03.018

Carattoli, A., Miriagou, V., Bertini, A., Loli, A., Colinon, C., Villa, L., et al. (2006). Replicon typing of plasmids encoding resistance to newer beta-lactams. Emerg. Infect. Dis. 12, 1145-1148. doi: 10.3201/eid1207.051555

Carattoli, A., Zankari, E., García-Fernández, A., Voldby Larsen, M., Lund, O., Villa, L., et al. (2014). In silico detection and typing of plasmids using PlasmidFinder and plasmid multilocus sequence typing. Antimicrob. Agents Chemother. 58, 3895-3903. doi: 10.1128/AAC. 02412-14

Castresana, J. (2000). Selection of conserved blocks from multiple alignments for their use in phylogenetic analysis. Mol. Biol. Evol. 17, 540-552. doi: 10.1093/ oxfordjournals.molbev.a026334

CLSI (2015). Performance Standards for Antimicrobial Susceptibility Testing; Twenty-Second Informational Supplement. Approved Standard M100-S25. Wayne: Clinical and Laboratory Standards Institute.

Conte, V., Monaco, M., Giani, T., D’Ancona, F., Moro, M. L., Arena, F., et al. (2016). Molecular epidemiology of KPC-producing Klebsiella pneumoniae from invasive infections in Italy: increasing diversity with predominance of the ST512 clade II sublineage. J. Antimicrob. Chemother. 71, 3386-3391. doi: 10.1093/jac/ dkw337 
Crivaro, V., Bagattini, M., Salza, M. F., Raimondi, F., Rossano, F., Triassi, M., et al. (2007). Risk factors for extended-spectrum beta-lactamase-producing Serratia marcescens and Klebsiella pneumoniae acquisition in a neonatal intensive care unit. J. Hosp. Infect. 67, 135-141. doi: 10.1016/j.jhin.2007. 07.026

Crivaro, V., Bogdanović, L., Bagattini, M., Iula, V. D., Catania, M., Raimondi, F., et al. (2015). Surveillance of healthcare-associated infections in a neonatal intensive care unit in Italy during 2006-2010. BMC Infect. Dis. 15:152. doi: 10.1186/s12879-015-0909-9

Del Franco, M., Paone, L., Novati, R., Giacomazzi, C. G., Bagattini, M., Galotto, C., et al. (2015). Molecular epidemiology of carbapenem resistant Enterobacteriaceae in Valle d'Aosta region, Italy, shows the emergence of KPC-2 producing Klebsiella pneumoniae clonal complex 101 (ST101 and ST1789). BMC Microbiol. 15:260. doi: 10.1186/s12866-015-0597-z

Di Pilato, V., Pollini, S., and Rossolini, G. M. (2014). Characterization of plasmid pAX22, encoding VIM- 1 metallo- $\beta$-lactamase, reveals a new putative mechanism of In70 integron mobilization. J. Antimicrob. Chemother. 69, 67-71. doi: $10.1093 /$ jac/dkt311

Diancourt, L., Passet, V., Verhoef, J., Grimont, P. A., and Brisse, S. (2005). Multilocus sequence typing of Klebsiella pneumoniae nosocomial isolates. J. Clin. Microbiol. 43, 4178-4182. doi: 10.1128/JCM.43.8.41784182

Edgar, R. C. (2004). MUSCLE: multiple sequence alignment with high accuracy and high throughput. Nucleic Acids Res. 32, 1792-1797. doi: 10.1093/nar/gkh340

Esteban-Cantos, A., Aracil, B., Bautista, V., Ortega, A., Lara, N., Saez, D., et al. (2017). The carbapenemase-producing Klebsiella pneumoniae population is distinct and more clonal than the carbapenem-susceptible population. Antimicrob. Agents Chemother. 61:e02520-16. doi: 10.1128/AAC. 02520-16

EUCAST (2016). European Committee on Antimicrobial Susceptibility Testing Breakpoint Tables for Interpretation of MICs and Zone Diameters Version 6.0, Valid from 2016-01-01. Available at: http://www.eucast.org/clinical_ breakpoints/

European Centre for Disease Prevention and Control [ECDC] (2012). Antimicrobial Resistance Surveillance in Europe 2011. Annual Report of the European Antimicrobial Resistance Surveillance Network (ears-net). Stockholm: ECDC.

Feil, E. J., Li, B. C., Aanensen, D. M., Hanage, W. P., and Spratt, B. G. (2004). eBURST: inferring patterns of evolutionary descent among clusters of related bacterial genotypes from multilocus sequence typing data. J. Bacteriol. 186, 1518-1530. doi: 10.1128/JB.186.5.1518-1530.2004

Francisco, A. P., Vaz, C., Monteiro, P. T., Melo-Cristino, J., Ramirez, M., and Carrico, J. A. (2012). PHYLOViZ: phylogenetic inference and data visualization for sequence based typing methods. BMC Bioinformatics 13:87. doi: 10.1186/ 1471-2105-13-87

Fricke, W. F., Welch, T. J., McDermott, P. F., Mammel, M. K., LeClerc, J. E., White, D. G., et al. (2009). Comparative genomics of the IncA/C multidrug resistance plasmid family. J. Bacteriol. 191, 4750-4757. doi: 10.1128/JB.00189-09

Gaiarsa, S., Comandatore, F., Gaibani, P., Corbella, M., Dalla Valle, C., Epis, S., et al. (2015). Genomic epidemiology of Klebsiella pneumoniae in Italy and novel insights into the origin and global evolution of its resistance to carbapenem antibiotics. Antimicrob. Agents Chemother. 59, 389-396. doi: 10.1128/AAC.04224-14

Giani, T., Antonelli, A., Caltagirone, M., Mauri, C., Nicchi, J., Arena, F., et al. (2017). Evolving beta-lactamase epidemiology in Enterobacteriaceae from Italian nationwide surveillance, October 2013: KPC-carbapenemase spreading among outpatients. Euro Surveill. 22:30583. doi: 10.2807/1560-7917.ES.2017. 22.31.30583

Goodman, K. E., Simner, P. J., Tamma, P. D., and Milstone, A. M. (2016). Infection control implications of heterogeneous resistance mechanisms in carbapenemresistant Enterobacteriaceae (CRE). Expert Rev. Infect. Ther. 14, 95-108. doi: 10.1586/14787210.2016.1106940

Grundmann, H., Glasner, C., Albiger, B., Aanensen, D. M., Tomlinson, C. T., Andrasević, A. T., et al. (2017). Occurrence of carbapenemaseproducing Klebsiella pneumoniae and Escherichia coli in the European survey of carbapenemase-producing Enterobacteriaceae (EuSCAPE): a prospective, multinational study. Lancet Infect. Dis. 17, 153-163. doi: 10.1016/S14733099(16)30257-2
Guindon, S., Dufayard, J. F., Lefort, V., Anisimova, M., Hordijk, W., and Gascuel, O. (2010). New algorithms and methods to estimate maximumlikelihood phylogenies: assessing the performance of PhyML 3.0. Syst. Biol. 59, 307-321. doi: 10.1093/sysbio/syq010

Hancock, S. J., Phan, M. D., Peters, K. M., Forde, B. M., Chong, T. M., Yin, W. F., et al. (2017). Identification of IncA/C plasmid replication and maintenance genes and development of a plasmid multi-locus sequence-typing scheme. Antimicrob. Agents Chemother. 61:e01740-16. doi: 10.1128/AAC.01740-16

Horan, T. C., Andrus, M., and Dudeck, M. A. (2008). CDC/NHSN surveillance definition of health care-associated infection and criteria for specific types of infections in the acute care setting. Am. J. Infect. Control 36, 309-332. doi: 10.1016/j.ajic.2008.03.002

Jacoby, G. A., and Han, P. (1996). Detection of extended-spectrum beta-lactamases in clinical isolates of Klebsiella pneumoniae and Escherichia coli. J. Clin. Microbiol. 34, 908-911.

Jolley, K. A., and Maiden, M. C. (2010). BIGSdb: scalable analysis of bacterial genome variation at the population level. BMC Bioinformatics 11:595. doi: 10.1186/1471-2105-11-595

Khan, A. U., Maryam, L., and Zarrilli, R. (2017). Structure, genetics and worldwide spread of New Delhi Metallo- $\beta$-lactamase (NDM): a threat to public health. BMC Microbiol. 17:101. doi: 10.1186/s12866-017-1012-8

Langmead, B. V., and Salzberg, S. (2012). Fast gapped-read alignment with Bowtie 2. Nat. Methods 9, 357-359. doi: 10.1038/nmeth.1923

Logan, L. K. (2012). Carbapenem-resistant enterobacteriaceae: an emerging problem in children. Clin. Infect. Dis. 55, 852-859. doi: 10.1093/cid/cis543

Logan, L. K., Renschler, J. P., Gandra, S., Weinstein, R. A., Laxminarayan, R., and Centers for Disease Control Prevention Epicenters Program (2015). Carbapenem-resistant Enterobacteriaceae in children, United States, 1999-2012. Emerg. Infect. Dis. 21, 2014-2021. doi: 10.3201/eid2111.150548

Logan, L. K., and Weinstein, R. A. (2017). The epidemiology of carbapenemresistant Enterobacteriaceae: the impact and evolution of a global menace. J. Infect. Dis. 215(Suppl. 1), S28-S36. doi: 10.1093/infdis/jiw282

Maatallah, M., Vading, M., Kabir, M. H., Bakhrouf, A., Kalin, M., Nauclér, P., et al. (2014). Klebsiella variicola is a frequent cause of bloodstream infection in the Stockholm area, and associated with higher mortality compared to K. pneumoniae. PLOS ONE 9:e113539. doi: 10.1371/journal.pone.0113539

Matsumura, Y., Peirano, G., Devinney, R., Bradford, P. A., Motyl, M. R., Adams, M. D., et al. (2017). Genomic epidemiology of global VIM-producing Enterobacteriaceae. J. Antimicrob. Chemother. doi: 10.1093/jac/dkx148 [Epub ahead of print].

Ministero della Salute (2013). Sorveglianza e Controllo delle Infezioni da Batteri Produttori di Carbapenemasi (CPE) (Circolare n 4968 26/02/2013). Available at http://www.salute.gov.it/portale/documentazione/p6_2_6.jsp?lingua= italiano\&area27\&btnCerca $=$ cerca\&iPageNo $=4$ [accessed November 6, 2015].

Nordmann, P., and Poirel, L. (2014). The difficult-to-control spread of carbapenemase producers among Enterobacteriaceae worldwide. Clin. Microbiol. Infect. 20, 821-830. doi: 10.1111/1469-0691.12719

Onori, R., Gaiarsa, S., Comandatore, F., Pongolini, S., Brisse, S., Colombo, A., et al. (2015). Tracking nosocomial Klebsiella pneumoniae infections and outbreaks by whole-genome analysis: small-scale Italian scenario within a single hospital. J. Clin. Microbiol. 53, 2861-2868. doi: 10.1128/JCM.00545-15

Paulin-Curlee, G. G., Singer, R. S., Sreevatsan, S., Isaacson, R., Reneau, J., Foster, D., et al. (2007). Genetic diversity of mastitis-associated Klebsiella pneumoniae in dairy cows. J. Dairy Sci. 90, 3681-3689. doi: 10.3168/jds.2006-776

Peirano, G., Lascols, C., Hackel, M., Hoban, D. J., and Pitout, J. D. (2014). Molecular epidemiology of Enterobacteriaceae that produce VIMs and IMPs from the SMART surveillance program. Diagn. Microbiol. Infect. Dis. 78, 277-281. doi: 10.1016/j.diagmicrobio.2013.11.024

Pitout, J. D., Nordmann, P., and Poirel, L. (2015). Carbapenemase-producing Klebsiella pneumoniae, a key pathogen set for global nosocomial dominance. Antimicrob. Agents Chemother. 59, 5873-5884. doi: 10.1128/AAC.01019-15

Poirel, L., Walsh, T. R., Cuvillier, V., and Nordmann, P. (2011). Multiplex PCR for detection of acquired carbapenemase genes. Diagn. Microbiol. Infect. Dis. 70, 119-123. doi: 10.1016/j.diagmicrobio.2010.12.002

Porres-Osante, N., Azcona-Gutiérrez, J. M., Rojo-Bezares, B., Undabeitia, E., Torres, C., and Sáenz, Y. (2014). Emergence of a multiresistant KPC-3 and VIM-1 carbapenemase-producing Escherichia coli strain in Spain. J. Antimicrob. Chemother. 69, 1792-1795. doi: 10.1093/jac/dku055 
Pournaras, S., Zarkotou, O., Poulou, A., Kristo, I., Vrioni, G., Themeli-Digalaki, K., et al. (2013). A combined disk test for direct differentiation of carbapenemaseproducing Enterobacteriaceae in surveillance rectal swabs. J. Clin. Microbiol. 51, 2986-2990. doi: 10.1128/JCM.00901-13

Riccio, M. L., Franceschini, N., Boschi, L., Caravelli, B., Cornaglia, G., Fontana, R., et al. (2000). Characterization of the metallo- $\beta$-lactamase determinant of Acinetobacter baumannii AC- 54/97 reveals the existence of blaIMP allelic variants carried by gene cassettes of different phylogeny. Antimicrob. Agents Chemother. 44, 1229-1235. doi: 10.1128/AAC.44.5.1229-1235.2000

Seemann, T. (2014). Prokka: rapid prokaryotic genome annotation. Bioinformatics 30, 2068-2069. doi: 10.1093/bioinformatics/btu153

Viau, R., Frank, K. M., Jacobs, M. R., Wilson, B., Kaye, K., Donskey, C. J., et al. (2016). Intestinal carriage of carbapenemase-producing organisms: current status of surveillance methods. Clin. Microbiol. Rev. 29, 1-27. doi: 10.1128/ CMR.00108-14

Wick, R. R., Schultz, M. B., Zobel, J., and Holt, K. E. (2015). Bandage: interactive visualization of de novo genome assemblies. Bioinformatics 31, 3350-3352. doi: 10.1093/bioinformatics/btv383

Yan, J. J., Zheng, P. X., Wang, M. C., Tsai, S. H., Wang, L. R., and Wu, J. J. (2015). Allocation of Klebsiella pneumoniae bloodstream isolates into four distinct groups by ompK36 typing in a Taiwanese university hospital. J. Clin. Microbiol. 53, 3256-3263. doi: 10.1128/JCM.01152-15

Zarrilli, R., Tripodi, M. F., Di Popolo, A., Fortunato, R., Bagattini, M., Crispino, M., et al. (2005). Molecular epidemiology of high-level aminoglycoside-resistant enterococci isolated from patients in a university hospital in southern Italy. J. Antimicrob. Chemother. 56, 827-835. doi: 10.1093/jac/dki347

Zhu, J., Ding, B., Xu, X., Zhu, D., Yang, F., Zhang, H., et al. (2015). Klebsiella pneumoniae: development of carbapenem resistance due to acquisition of bla NDM-1 during antimicrobial therapy in twin Infants with pneumonia. Front. Microbiol. 6:1399. doi: 10.3389/fmicb.2015. 01399

Zurfluh, K., Power, K. A., Klumpp, J., Wang, J., Fanning, S., and Stephan, R. (2015). A novel Tn3-like composite transposon harboring blaVIM-1 in Klebsiella pneumoniae spp. pneumoniae isolated from river water. Microb. Drug Resist. 21, 43-49. doi: 10.1089/mdr.2014. 0055

Conflict of Interest Statement: The authors declare that the research was conducted in the absence of any commercial or financial relationships that could be construed as a potential conflict of interest.

Copyright (c) 2017 Esposito, Gaiarsa, Del Franco, Crivaro, Bernardo, Cuccurullo, Pennino, Triassi, Marone, Sassera and Zarrilli. This is an open-access article distributed under the terms of the Creative Commons Attribution License (CC BY). The use, distribution or reproduction in other forums is permitted, provided the original author(s) or licensor are credited and that the original publication in this journal is cited, in accordance with accepted academic practice. No use, distribution or reproduction is permitted which does not comply with these terms. 